MATEC Web of Conferences 22,05014 (2015)

DOI: $10.1051 /$ matecconf/ 20152205014

(C) Owned by the authors, published by EDP Sciences, 2015

\title{
Biomechanical Analysis of Contemporary Throwing Technique Theory
}

Jian Chen

Department of Physical Education, Ocean University of China, Qingdao, Shandong, China

\begin{abstract}
Based on the movement process of throwing and in order to further improve the throwing technique of our country, this paper will first illustrate the main influence factors which will affect the shot distance via the mutual combination of movement equation and geometrical analysis. And then, it will give the equation of the acting force that the throwing athletes have to bear during throwing movement; and will reach the speed relationship between each arthrosis during throwing and batting based on the kinetic analysis of the throwing athletes' arms while throwing. This paper will obtain the momentum relationship of the athletes' each arthrosis by means of rotational inertia analysis; and then establish a restricted particle dynamics equation from the Lagrange equation. The obtained result shows that the momentum of throwing depends on the momentum of the athletes' wrist joints while batting.
\end{abstract}

Keywords: analysis of throwing technique; mechanical analysis; biodynamic analysis; rotational inertia and tensor

\section{INTRODUCTION}

As the times progress, the technologies of track \& field and throwing sports have been gradually improved and obtained great breakthrough. The current world records have exceeded the previous ones by a great extent. During the development of throwing in China, the achievement had never been good before the founding of New China. With the founding of New China, our nation has been liberated and our strength in each sport has achieved remarkably improvement. After the establishment of the reform and opening-up policy, China's comprehensive national strength has been in continuous rise and our status in sports field is becoming increasingly higher. Our enhanced learning and mastering of sport techniques have laid a solid foundation for the leap in our throwing field. In order to further improve our techniques in throwing, this paper will analyze the influence that shot speed and shot angle have on throwing performance according to the movement process of throwing. It will also offer the study and analysis of the impact that human biomechanics has on the shot speed and angle of release, so as to provide better corresponding exercise for the athletes and help them improve their performance.

\section{ESTABLISHMENT AND SOLVING OF MODEL}

\subsection{Principle of Throwing}

The movement process of throwing can be divided into two parts: after the shot and before the shot. Before the shot, the thrown object gets initial velocity from the athlete's own movement. After the shot, the thrown object moves in parabolic motion that only bears gravity effect. The final result is related to the shot speed, the angle of release and the shot height of the weight throw. Therefore, if a throwing athlete wants to get ideal performance, he/she must find the best shot height and angle of release. The distance between the shot spot and the landing spot of the thrown object is composed of two parts: distance before the shot and distance after the shot. The factors influencing these two parts are shown in Figure 1 below.

The thrown weight throw only does parabolic motion after the shot due to gravity effect. The equation is as follows:

$$
\left\{\begin{array}{l}
x=v_{0 x} \cdot t=v_{0} \cos \theta \cdot t \\
y=v_{0 y} \cdot t-\frac{1}{2} g t^{2}=v_{0} \sin \theta \cdot t-\frac{1}{2} g t^{2}
\end{array}\right.
$$

The equation of $t$ can be solved by $y$ and can be brought into the equation above to obtain the following calculating equation of throwing distance:

$x=\frac{v_{0}^{2} \sin \theta \cos \theta+v_{0} \cos \theta\left(v_{0}^{2} \sin ^{2} \theta+2 g h\right)^{1 / 2}}{g}$

It can be seen from the calculating equation of throwing result that the angle of release, shot height and final shot speed can leave great influence on the throwing distance among which the final shot speed is the first influencing factor.

\subsection{Analysis of the Final Shot Speed}

This paper will first analyze the influence that the final shot speed has on the final result with the condition that both the angle of release and shot height are fixed. 


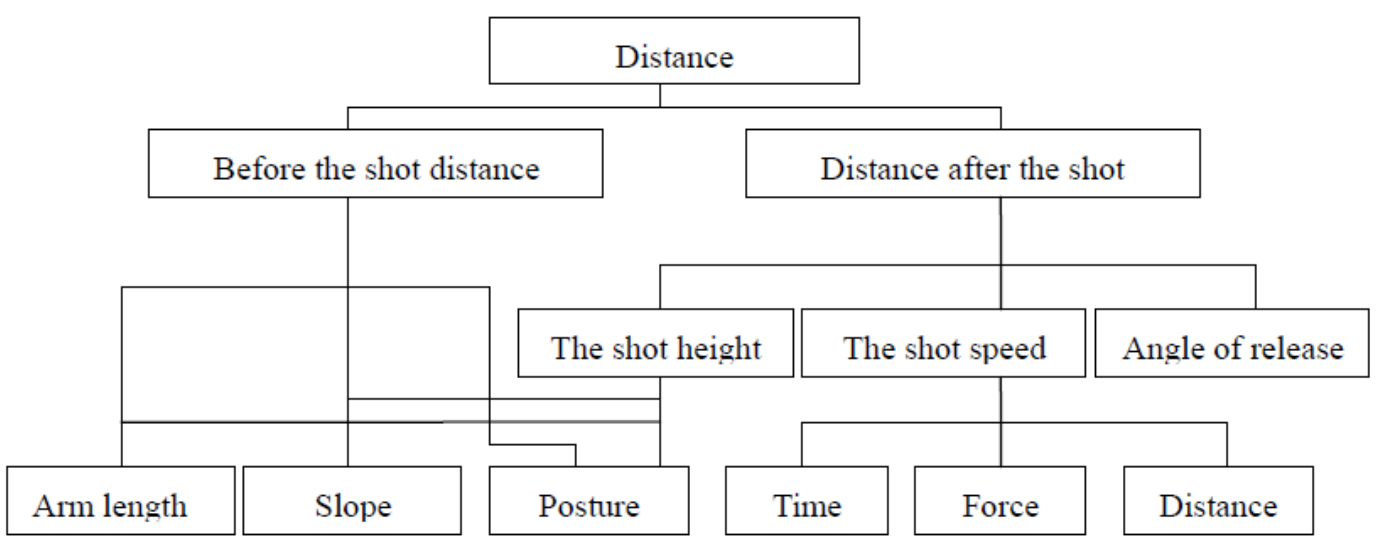

Figure 1. Analysis of the Influence Factors of Distance

Table 1. The Influence that the Final Shot Speed Has on the Result

\begin{tabular}{llllllllllll}
\hline Speed $(\mathrm{m} / \mathrm{s})$ & 14 & 20 & 22 & 23 & 24 & 25 & 26 & 27 & 28 & 29 & 30 \\
\hline Result $(m)$ & 25.45 & 42.57 & 50.83 & 55.46 & 60.03 & 65.12 & 70.24 & 82.31 & 87.88 & 93.4 & 97.5
\end{tabular}

Table 2. The Influence that Different Angles of Release Have on the Distance

\begin{tabular}{llllllllllll}
\hline Angle & 30 & 35 & 38 & 40 & 43 & 45 & 48 & 50 & 55 & 58 & 60 \\
\hline Distance $(\mathrm{m})$ & 66.92 & 71.98 & 74.05 & 75.00 & 75.78 & 75.86 & 78.63 & 74.89 & 70.68 & 68.31 & 65.42 \\
\hline
\end{tabular}

Fixed parameters: the angle of release is $43^{\circ}$ and the shot height is $1.6 \mathrm{~m}$. See Table 1 for the results.

It can be seen from the table above that the final shot speed has obvious influence on the throwing results. When the speed is $14 \mathrm{~m} / \mathrm{s}$, the throwing distance is about $25 \mathrm{~m}$. However, when the final shot speed of an ordinary athlete is within $26 \sim 30 \mathrm{~m} / \mathrm{s}$, the throwing distance can be $70 \mathrm{~m}$ if the shot speed can reach $26 \mathrm{~m} / \mathrm{s}$. When the final shot speed is $27 \mathrm{~m} / \mathrm{s}$ or $28 \mathrm{~m} / \mathrm{s}$, the throwing distance can be $80 \mathrm{~m}$ or even $90 \mathrm{~m}$. As a result, the improvement of the final shot speed can lead to the improvement in the athletes' final throwing performance.

\subsection{Analysis of the Angle of Release and the Shot Height}

When there's little air friction, the movement of the thrown weight throw can be regarded as standard oblique projectile motion. Set the speed and the initial spot as constant values, different angles of release can make large differences in the athletes' throwing results. See the parabolic paths formed by different angles of release in Figure 2.

It can be seen from Figure 2 that the angle of release can only lead to the best distance within a certain range. When there's no influence from air friction, the parabola span can reach its maximum value if the angle of release is $45^{\circ}$. Take weight throw as an ex- ample; and see the relationship between the angle of release and the distance in Table 2.

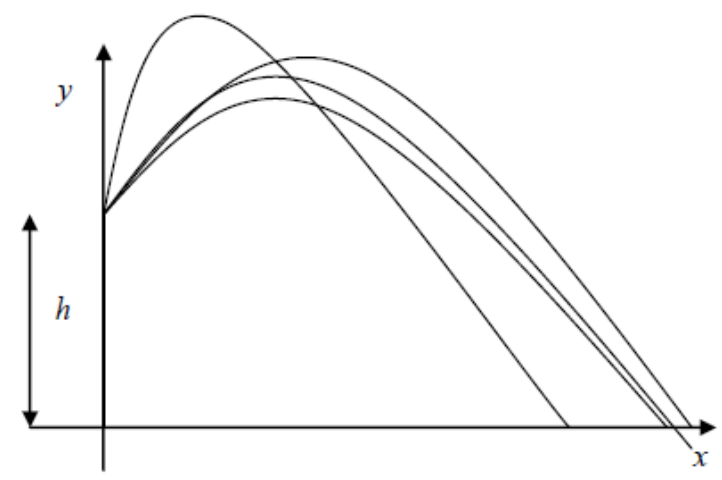

Figure 2. The relation between angle of release and distance

In the physical situation, besides the gravity effect, the weight throw will also be affected by the resistance formed in air streaming. For simplification, regard the weight throw as a material point. In accordance with Newton's laws of motion, the motion mode of the weight throw is shown in Figure 3. 


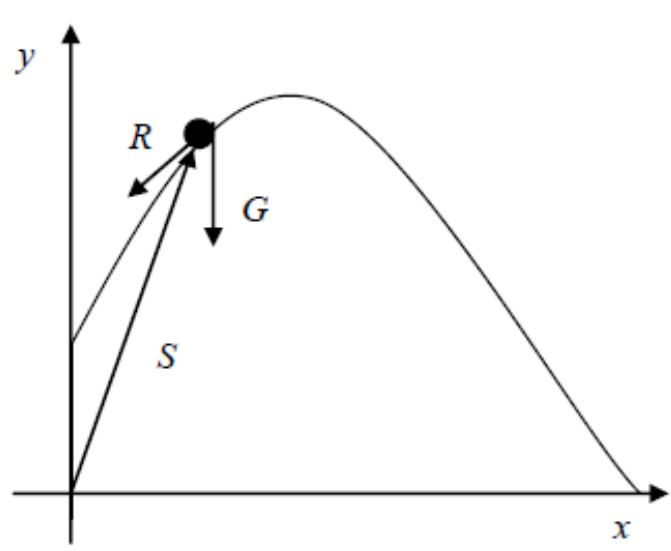

Figure 3. The Schematic Diagram of the Weight Throw Movement in the Air

In the figure, $S$ refers to the radius vector while $R$ refers to the air friction. Therefore, the weight throw movement can be expressed by the differential equation shown as follows:

$m \frac{d^{2} s}{d t^{2}}=G+R$

According to hydromechanics, we also know that wake flow will appear when the weight throw flies in the air, and it will form streaming resistance to the air. The streaming resistance and the air dynamical pressure are in direct proportion. The expression is given below:

$R=-c A \frac{\rho V^{2}}{2}$

Among which, c refers to the resistance coefficient; $A=\frac{\pi d^{2}}{4} ; \rho$ refers to the air density; $v$ refers to the speed of the weight throw; and $d$ refers to the diameter of the weight throw. The equation set given below can be obtained by bringing equation (2) into equation (1):

$$
\left\{\begin{array}{c}
m \frac{d^{2} x}{d t^{2}}=-\frac{c A \rho}{2}\left(v_{x}^{2}+v_{y}^{2}\right)^{\frac{1}{2}} v_{x} \\
m \frac{d^{2} y}{d t^{2}}=-m g-\frac{c A \rho}{2}\left(v_{x}^{2}+v_{y}^{2}\right)^{\frac{1}{2}} v_{y}
\end{array}\right.
$$

While solving the above equation set, set $z_{1}=x, z_{2}=\frac{d x}{d t}, z_{3}=y, z_{4}=\frac{d y}{d t}$ in order to simplify

the above equation set into four differential equations of first order:

$$
\left\{\begin{array}{l}
\frac{d z_{1}}{d t}=z_{2} \\
\frac{d z_{2}}{d t}=-\frac{c A \rho}{2 m}\left(z_{2}^{2}+z_{4}^{2}\right)^{\frac{1}{2}} z_{2} \\
\frac{d z_{3}}{d t}=z_{4} \\
\frac{d z_{4}}{d t}=-g-\frac{c A \rho}{2 m}\left(z_{2}^{2}+z_{4}^{2}\right)^{\frac{1}{2}} z_{4}
\end{array}\right.
$$

Apply Runge-kutta numerical integration to solve the above equations and obtain the relationship between the flying distance of the weight throw and the angle of release, the shot height, the shot speed and the air friction coefficient. The results show that the angle of release, the shot height, the final shot speed and the air friction coefficient can leave different influences on the athletes' final performance. And among the influence factors, the final shot speed has the greatest influence. With the increasing of the final shot speed, the flying distance of the weight throw can be greatly improved. However, the influences that the shot height and the air friction coefficient have on the athletes' final performance are not so obvious. In the physical condition, the athletes stand in the basically same environment and share the same average height As a result, the best choice to improve the throwing performance is to enhance the training for the final shot speed and the control of the angle of release.

\subsection{Kinetic Analysis of Throwing}

Regard the upper arm and the forearm that an athlete uses while throwing as two rigid bodies with different volumes; and integrate the shoulder joint, elbow joint and wrist joint into a model with three degrees of freedom as shown in Figure 4.

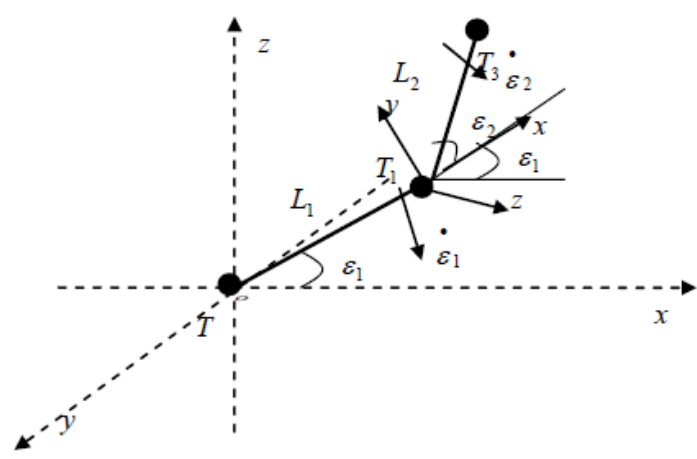

Figure 4. The Schematic Diagram of the Degrees of Freedom of the Throwing Athlete's Throwing Arm

Set the throwing athlete's shoulder joint, elbow 
joint and wrist joint as $T, T_{1}$ and $T_{3}$ respectively; set the upper arm and the forearm as $L_{1}, L_{2}$; and set the dissection angles as $\varepsilon_{1}, \varepsilon_{2}$. Among which, the three dimensional vectors of $T$ and $T_{1}$ are $\dot{\gamma}_{1}, \dot{\gamma}_{2}$ acting as the angular velocities of the athlete's throwing upper arm and forearm relative to the earth. The speed of $T_{1}$ is $\dot{\varepsilon}_{2}$ and its value can be calculated as follows:

$$
\dot{\varepsilon}_{2}=\dot{\gamma}_{2}-\dot{\gamma}_{1}
$$

When a throwing athlete does throwing, the shot speed and the momentum of the thrown object depend on the speed of the wrist at the moment of throwing. However, the angle speeds of the upper arm and the forearm which do the throwing motion and the angle speed of $T_{1}$ can leave influence on the angle speed of $T_{3}$. As $T_{1}$ leads $L_{2}$ to do translation and rotation during its circling motion, the speed of $T_{3}$ and its relative speed is related to the speed of $T_{1}$, which is:

$$
\vec{C}\left(\vec{T}_{1}\right)_{G}=\dot{\varepsilon}_{1} \times \vec{R}_{1}=\dot{\varepsilon}_{1} \times \vec{R}_{1}
$$

$C\left(\vec{T}_{3}\right)_{L}=\dot{\varepsilon}_{2} \times \vec{R}_{2}$

$$
\vec{C}\left(\vec{T}_{1}\right)_{G} \text { refers to the velocity vector of } T_{1} \text { in the }
$$
reference system and $C\left(\vec{T}_{3}\right)_{L}$ refers to the speed of $T_{3}$ relative to $T_{1} \cdot \dot{\varepsilon}_{1}, \dot{\varepsilon}_{2}$ respectively refer to the angle speeds of $T$ and $T_{1} . \quad \vec{R}_{1}$ refers to the position vectors form $T$ to $T_{1}$ while $\vec{R}_{2}$ refers to the position vectors from $T_{1}$ to $T_{3}$.

In order to obtain the speed of $T_{3}$ which is relative to the reference system: $\dot{T}_{3 G}=\vec{C}\left(\vec{T}_{3}\right)_{G}$, the influence that the local motion of $L_{1}$ and $L_{2}$ has on $T_{1}$ should be first calculated. According to the theorem of vector, the following equation can be obtained:

$\dot{T}_{3 G}=\dot{\varepsilon}_{1} \times \vec{R}_{1}+\dot{\varepsilon}_{2} \times \vec{R}_{2}+\dot{\varepsilon}_{1} \times \vec{R}_{2}$

$\dot{T}_{3 G}=\dot{\varepsilon}_{1}\left(\vec{R}_{1}+\vec{R}_{2}\right)+\dot{\varepsilon}_{2} \times \vec{R}_{2}$

It can be simplified as:

$$
\dot{T}_{3_{G}}=\vec{T}_{3_{G}} \times \dot{\varepsilon}_{1}+\dot{\varepsilon}_{2} \times \vec{R}_{2}
$$

$\vec{T}_{3 G} \times \dot{\varepsilon}_{1}$ refers to the speed of $T_{1}$ generated by $T$; $\dot{\varepsilon_{2}} \times \vec{R}_{2}$ refers to the speed of $T_{3}$ generated by $T_{1}$; $T_{3_{G}}$ refers to the position vector of $T_{3}$ in the reference system.

In order to express the speed relation among $T, T_{1}$ and $T_{3}$ more specifically, the relationship between the angles of $T$ and $T_{1}$ with the position of $T_{3}$ in the model shown in Figure 3 can be written as:

$\left\{\begin{array}{l}x_{p}=R_{1} \cos \varepsilon_{1}+R_{2} \cos \left(\varepsilon_{1}+\varepsilon_{2}\right) \\ y_{p}=R_{1} \sin \varepsilon_{1}+R_{2} \sin \left(\varepsilon_{1}+\varepsilon_{2}\right) \\ z_{p}=R_{1} \cos \varepsilon_{1}+R_{2} \sin \left(\varepsilon_{1}+\varepsilon_{2}\right)\end{array}\right.$

Conduct differential operation to the angles of $T$ and $T_{1}$. The relationship between the angles of $T$ and $T_{1}$ with the position of $T_{3}$ can be obtained from the equation above:

$$
\left\{\begin{array}{c}
d X=\frac{\partial X\left(\varepsilon_{1}, \varepsilon_{2}\right)}{\partial \varepsilon_{1}} d \varepsilon_{1}+\frac{\partial X\left(\varepsilon_{1}, \varepsilon_{2}\right)}{\partial \varepsilon_{2}} d \varepsilon_{2} \\
d Y=\frac{\partial Y\left(\varepsilon_{1}, \varepsilon_{2}\right)}{\partial \varepsilon_{1}}+\frac{\partial Y\left(\varepsilon_{1}, \varepsilon_{2}\right)}{\partial \varepsilon_{2}} d \varepsilon_{2} \\
d Z=\frac{\partial Z\left(\varepsilon_{1}, \varepsilon_{2}\right)}{\partial \varepsilon_{1}}+\frac{\partial Z\left(\varepsilon_{1}, \varepsilon_{2}\right)}{\partial \varepsilon_{2}} d \varepsilon_{2}
\end{array}\right.
$$

Converse the equation above into matrix form as:

$\left(\begin{array}{l}d X \\ d Y \\ d Z\end{array}\right)=\left(\begin{array}{ll}\frac{\partial X\left(\varepsilon_{1}, \varepsilon_{2}\right)}{\partial \varepsilon_{1}} & \frac{\partial X\left(\varepsilon_{1}, \varepsilon_{2}\right)}{\partial \varepsilon_{2}} \\ \frac{\partial Y\left(\varepsilon_{1}, \varepsilon_{2}\right)}{\partial \varepsilon_{1}} & \frac{\partial Y\left(\varepsilon_{1}, \varepsilon_{2}\right)}{\partial \varepsilon_{2}} \\ \frac{\partial Z\left(\varepsilon_{1}, \varepsilon_{2}\right)}{\partial \varepsilon_{1}} & \frac{\partial Z\left(\varepsilon_{1}, \varepsilon_{2}\right)}{\partial \varepsilon_{2}}\end{array}\right)\left(\begin{array}{l}d \varepsilon_{1} \\ d \varepsilon_{2}\end{array}\right)$

According to the nature of the matrix and the vector product method, the equation above can be written as: $d \vec{T}_{3 G}=\vec{Y} d \vec{\varepsilon}$, among which $\vec{Y}$ is

$$
\vec{Y}=\left(\begin{array}{ll}
\frac{\partial X}{\partial \varepsilon_{1}} & \frac{\partial X}{\partial \varepsilon_{2}} \\
\frac{\partial Y}{\partial \varepsilon_{1}} & \frac{\partial Y}{\partial \varepsilon_{2}} \\
\frac{\partial Z}{\partial \varepsilon_{1}} & \frac{\partial Z}{\partial \varepsilon_{2}}
\end{array}\right)
$$

$\vec{Y}$ refers to the differential relationship between the angular displacement of the node in the current structure and the infinitesimal displacement of $T_{3}$. The following equation can be obtained by bringing the matrix relation equation into the equation above: 
$\frac{d \vec{T}_{3_{G}}}{d t}=\vec{Y} \frac{d \vec{\varepsilon}}{d t}$ or $\vec{T}_{3 G}=\vec{Y}\left[\dot{\varepsilon}_{1}, \dot{\varepsilon}_{2}\right]^{T}$

The following equation can be obtained by bringing the equation above into the calculating equation for the relative speed of $T_{3}$ :

$$
\dot{O}_{G}=\left(\begin{array}{ll}
\frac{\partial X}{\partial \varepsilon_{1}} & \frac{\partial X}{\partial \varepsilon_{2}} \\
\frac{\partial Y}{\partial \varepsilon_{1}} & \frac{\partial Y}{\partial \varepsilon_{2}} \\
\frac{\partial Z}{\partial \varepsilon_{1}} & \frac{\partial Z}{\partial \varepsilon_{2}}
\end{array}\right)\left[\vec{\varepsilon}_{1}, \vec{\varepsilon}_{2}\right]^{T} \dot{\varepsilon}_{1}+\dot{\varepsilon}_{2} \times \vec{R}_{2}
$$

According to the rotational inertia principle, the overall rotational inertia of the throwing athlete should be:

$$
P=\Sigma M_{i} H_{i}^{2}
$$

Among which, $M_{i}$ refers to the quality of each particle of a human body and $H$ refers to the distance from the athlete's each particle to the axis. As a human body is a rigid body with continuous distribution, the following equation can be obtained:

$P=\iiint_{v} H^{2} d m=\iiint_{v} H^{2} \rho d V$

$\leftrightarrow \rho$ refers to human density. The rotational tensor $\stackrel{\leftrightarrow}{Y}_{c}$ of the arm that the throwing athlete uses while throwing is as follows:

$\stackrel{\leftrightarrow}{Y}_{c}=\iiint_{v} \rho\left(H^{2} \stackrel{\leftrightarrow}{E}-\vec{H} \vec{H}\right) d V$

The expression of the vector quantity on the arbitrary point of the throwing athlete's body is $\vec{H}=H_{1} \vec{E}_{1}+H_{2} \vec{E}_{2}+H_{3} \vec{E}_{3} \cdot \vec{H} \vec{H}$ refers to the product of two vectors. The unit tensor is:

$\stackrel{\leftrightarrow}{E}=\left[\begin{array}{lll}1 & & \\ & 1 & \\ & & 1\end{array}\right]$

The unit orthogonal curve frame is $\left(C ; \vec{E}_{1}, \vec{E}_{2}, \vec{E}_{3}\right)$.

The resultant moment of the arm that the throwing athlete uses while throwing is $\Sigma \vec{k}_{c} . \quad \vec{\beta}$ refers to the athlete's acceleration vector in the inertia moving system while $\vec{\omega}$ refers to angular speed. Therefore, the rotational tensor moment equation of the athlete's arm while throwing is as follows:

$\sum \vec{k}_{c}=\stackrel{\leftrightarrow}{Y}_{c} \cdot \vec{\beta}+\vec{\omega} \times \overleftrightarrow{Y}_{c} \cdot \vec{\beta}$
The moment equation above of the athlete's arm on each coordinate orientation is the projection of the original moment equation on the three-position coordinate system. Hence, the resultant external moment $T_{1}$ generated by the athlete's arm rotating while throwing is as follows:

$T_{1}=\eta_{1} \cdot J_{1}$

$\eta_{1}$ refers to the angular accelerated speed of the arm for throwing while $J_{1}$ refers to the rotational inertia of the arm:

$J_{1}=\frac{m_{1} j_{1}^{2}}{2}$

$j_{1}$ refers to the radius of the upper arm; $m_{1}$ refers to the quality of the upper arm; and $\eta_{1}$ refers to the angular accelerated speed of the upper arm:

$\eta_{1}=\frac{d w_{1}}{d t}=\frac{d^{2} \beta_{1}}{d t^{2}}$

Then, the angular speed of the forearm $\eta_{2}$ should be:

$\eta_{2}=\frac{d w_{2}}{d t}+\frac{d w_{1}}{d t}=\frac{d^{2} \beta_{2}}{d t^{2}}+\frac{d^{2} \beta_{1}}{d t^{2}}$

Establish a restricted particle dynamics equation according to the Lagrange equation. The $d$ Lagrange function $U$ is the difference value between the system kinetic energy $J$ and the potential energy $Q$ :

$U=J-Q$

The system dynamics equation should be:

$F_{i}=\frac{d}{d t}\left(\frac{\partial U}{\partial q_{i}}-\frac{\partial U}{\partial q_{i}}\right) \quad i=1,2, \mathrm{~L}, n$

Among which, $\stackrel{\&}{q}$ i refers to the corresponding speed of particle; $q_{i}$ refers to the kinetic energy of particle and the coordinate of potential energy; and $F_{i}$ refers to the force placed on the No. i coordinate. The included angles between the upper arm and the coordinate axis and between the forearm and the coordinate axis are $\varepsilon_{1}, \varepsilon_{2}$ respectively while the lengths are $L_{1}, L_{2}$ respectively. $p_{1}, p_{2}$ respectively refer to the distance from the centre-of-gravity positions of the upper arm and the forearm to the center of $T$ and the distance from the centre-of-gravity positions of the upper arm and the forearm to $T_{1}$. Thus, the barycentric coordinates of the upper arm $\left(X_{1}, Y_{1}\right)$ should be: $\left\{\begin{array}{l}X_{1}=p_{1} \sin \varepsilon_{1} \quad Y_{1}=p_{1} \cos \varepsilon_{1} \\ X_{2}=L_{1} \sin \varepsilon_{1}+p_{2} \sin \left(\varepsilon_{1}+\varepsilon_{2}\right) \quad Y_{2}=-L_{1} \cos \varepsilon_{1}-p_{2} \cos \left(\varepsilon_{1}+\varepsilon_{2}\right)\end{array}\right.$ 
In a similar way, the barycentric coordinates of the forearm $\left(X_{2}, Y_{2}\right)$ can also be calculated.

The expressions of the system kinetic energy $E_{k}$ and the system potential energy $E_{p}$ are as follows:

$$
\left\{\begin{array}{l}
E_{k}=E_{k 1}+E_{k 2}, E_{k 1}=\frac{1}{2} m_{1} p_{1}^{2} \varepsilon_{1}^{2} \\
E_{k 2}=\frac{1}{2} m_{2} L_{1}^{2} \varepsilon_{1}^{2}+\frac{1}{2} m_{2} p_{2}^{2}\left(\varepsilon_{1}+\varepsilon_{2}\right)^{2}+m_{2} L_{2} p_{2}\left(\varepsilon_{01}^{2}+\varepsilon_{1} \varepsilon_{2}\right) \cos \varepsilon_{2} \\
E_{p}=E_{p 1}+E_{p 2}, E_{p 1}=\frac{1}{2} m_{1} g p_{1}\left(1-\cos \varepsilon_{1}\right) \\
E_{p 2}=m_{2} g p_{2}\left[1-\cos \left(\varepsilon_{1}+\varepsilon_{2}\right)\right]+m_{2} g L_{1}\left(1-\cos \varepsilon_{1}\right)
\end{array}\right.
$$

The moment $M_{h}$ at $T$ and the moment $M_{k}$ at $T_{1}$ obtained by the Lagrange system kinetic equation are as follows:

The $B_{i j k}$ in the equation above is:

$$
\begin{aligned}
& B_{111}=0 \quad B_{222}=0 \quad B_{121}=0 \quad B_{22}=m_{2} p_{2}^{2} \\
& B_{11}=m_{1} p_{1}^{2}+m_{2} p_{2}^{2}+m_{2} L_{1}^{2}+2 m_{2} L_{1} p_{2} \cos \varepsilon_{2} \\
& B_{1}=\left(m_{1} p_{1}+m_{2} L_{1}\right) g \sin \varepsilon_{1}+m_{2} p_{2} g \sin \left(\varepsilon_{1}+\varepsilon_{2}\right) \\
& B_{12}=m_{2} p_{2}^{2}+m_{2} L_{1} p_{2} \cos \varepsilon_{2} \quad B_{21}=m_{2} p_{2}^{2}+m_{1} L_{1} p_{2} \cos \varepsilon_{2} \\
& B_{122}=-m_{2} L_{1} p_{2} \sin \varepsilon_{2} \quad B_{211}=m_{2} L_{1} p_{2} \sin \varepsilon_{2} \\
& \left.B_{112}=-2 m_{2} L_{1} p_{2} \sin \varepsilon_{2} \quad B_{212}=B_{122}+B_{211} \quad B_{2}=m_{2} p_{2} g \sin \left(\varepsilon_{1}+\varepsilon_{2}\right)\right]
\end{aligned}
$$

According to the analysis, it can be concluded that the momentum of throwing depends on the momentum of wrist while throwing. Therefore, while throwing, the athletes must enhance their arm swing dynamics. When $\varepsilon_{1}, \varepsilon_{2}$ accord with $38^{\circ}<\varepsilon^{1}+\varepsilon^{2}<95^{\circ}$ and $0<\dot{\varepsilon}_{1}<\dot{\varepsilon}_{2}$, the speed of $T_{3}$ can reach the maximum value on the sagittal surface. Furthermore, with the increasing of $\dot{\varepsilon}_{1}$ and $\dot{\varepsilon}_{2}$, the change rate of the dissection angles at $L_{1}$ and $L_{2}$ in unit time will reach the maximum values during the athlete's throwing. In this period, the change rate of the dissection angle at $L_{2}$ is greater than that of $L_{1}$ The principle is that the included angle of $L_{1}$ and $L_{2}$ at the moment of batting can be almost $180^{\circ}$, and the force will pass along the axis of $L_{1}$ to $L_{2}$. However, as $L_{1}$ and $L_{2}$ are connected with each other, there's a certain loss of the force during the process of passing. As a result, the angle speed at $L_{2}$ is greater than that of $L_{1}$ which is more beneficial for the acceleration at $T_{1}$. According to the kinetic analysis results of the athlete's arm while throwing, it can be concluded that during throwing, the throwing athletes should firstly adjust the angles of their shoulder joints to the minimum value; and then adjust the angles of elbow joints and wrist joints to the minimum values. Greater crook degree of arm can be more helpful to accelerate the speed of wrist joint and make the angle speed of forearm become greater than the angle speed of upper arm, so as to increase the mechanical impulse generated by batting.

\section{CONCLUSION}

This paper first obtains the main influence factors affecting the throwing distance by the mutual combination of motion equation and geometrical analysis. And then, it gives the acting force equation that the athletes have to bear during throwing. This paper also takes the influence that the air friction has on the throwing distance and the shot height into consideration. It establishes the kinetic differential equation of the weight throwing while flying in the air; and obtains the best angle of release.

Moreover, this paper also builds the speed relationship between each arthrosis during throwing and batting based on the kinetic analysis of the throwing athletes' arms while throwing. It obtains the momentum relationship of the athletes' each arthrosis by means of rotational inertia analysis; and then establishes a restricted particle dynamics equation from the Lagrange equation. The obtained result shows that the momentum of throwing depends on the momentum of the athletes' wrist joints while batting. Besides, while throwing, the athletes should first adjust the angles of their shoulder joints to the minimum value; and then adjust the angles of elbow joints and wrist joints to the minimum values. Greater crook degree of arm can be more helpful to accelerate the speed of wrist joint and make the angle speed of forearm become greater than the angle speed of upper arm, so as to increase the mechanical impulse generated by batting. The results show that the best angle of release should be $44^{\circ}$. When an athlete's final shot speed reaches $26 \sim 30 \mathrm{~m} / \mathrm{s}$, the acting force he/she bears is between $2 \mathrm{KN}$ and $3 \mathrm{KN}$. Besides, final shot speed can bring better performance. In professional games, the athletes will have the same average height and will be in the basically same environment. As a result, the influence that the shot height and the air friction have on the final performance is minor and can be neglected.

\section{REFERENCES}

[1] Theoretical Mechanics Teaching and Research Office of Harbin Institute of Technology. 2002. Theoretical Mechanics. Beijing: Higher Education Press.

[2] Prandtl L, Auswatiki K \& Vigerhart K. 1981. Introduction to Hydromechanics. Translated by Guo Y.H. \& Lu S.J. Beijing: Science Press.

[3] Li Y.G. 2003. Biomechanics Foundation of Exercise Training. Wuhan: Hubei Science and Technology Press, pp: 10.

[4] Vladimir. 2004. Translated by Lu A.Y. Sports Biomechanics. Beijing: People's Sports Publishing House of China, pp: 377-379.

[5] Drafting Group of Sports Biomechanics. 2000, Sports Biomechanics. Beijing: Higher Education Press, pp: 54.

[6] Wang Q. 2004. Application of sports biomechanics methods in analysis of motion. Journal of Shenyang Sport University, 2.

[7] Yan H.G. 2012. Sports Biomechanics. Beijing: Beijing Normal University Press. 
ICETA 2015

[8] Zhou Y. B. 2008. Theoretical Mechanics Course. Beijing: Higher Education Press, pp: 164-165. 